\title{
NONLINEAR DISPERSIVE INSTABILITIES IN MAGNETIC FLUIDS*
}

\author{
BY \\ S. K. MALIK AND M. SINGH \\ Simon Fraser University
}

\begin{abstract}
An asymptotic nonlinear theory of the two superposed magnetic fluids is presented taking into account the spatial as well as temporal effects. A generalized formulation of the evolution equation governing the amplitude is developed which leads to the nonlinear Klein-Gordon equation. The various stability criteria are derived from this equation. Obtained also are the bell shaped soliton and the kink solutions.
\end{abstract}

1. Introduction. In recent years, the nonlinear instability of two superposed ferrofluids has received considerable attention of various authors [1]-[4]. The magnetic fluids are synthesized in the laboratory and consist of ordinary nonconducting liquids in which very fine small particles (mean diameter varying rom 30 to $150^{\circ} \mathrm{A}$ ) of ferromagnetic material are suspended freely. The very small size of these suspended particles, which are distributed uniformly and homogeneously in the fluid, prevents coagulation. The introduction of an applied electric or a magnetic field does not cause the separation of the magnetic particles from the liquid. Upon switching off the field, the fluid fully recovers its original characteristics. The magnetic fluid is assumed to be non-conducting, and the only forces involved are due to polarization. Cowley and Rosensweig [1] have demonstrated that an instability sets in when the applied magnetic field $H$, which is normal to the fluid surface, is slightly greater than the critical magnetic field $H_{c}$. It was reported in their experiments that such an instability results in the appearance of the regular hexagonal cells on the fluid surface. This experimental observation has been theoretically studied by Gailitis [5], Kuznetsov and Spektor [6], and Brancher [7] with the use of the energy method. Gailitis [5] has demonstrated the existence of hard excitation of the steady waves, and shown that for certain values of the magnetic field strength, the hexagonal cell is replaced by a square cell with possible hystersis behaviour for the subcritical values of the field. Based on bifurcation analysis, Twombly and Thomas [8] have also obtained similar results.

The aim of this presentation is to examine how a continuous band width of the modes affects the description of the post critical instability. The method we employ was developed by Stuart [9], and later modified by Newell [10] to consider the development of the waves at the critical or the bifurcation point. In Sec. 2, we formulate the problem, give

\footnotetext{
* Received by the editors September 7, 1983.
} 
the basic equations, and review the linear theory governing the wave motion on the surface of two superposed magnetic fluids. With the use of the multiple scales method, we show in Sec. 3 that the evolution of the small but finite amplitude wave-pocket is governed by the nonlinear Klein-Gordon equation. Depending upon the value of the magnetic field strength $H$ and the magnetic permeabilities of the media involved, this equation gives exponential decay, subcritical and explosive instabilities if only temporal variations are taken into consideration. The nonlinear Klein-Gordon equation has a wave train solution in terms of the Jacobian elliptic function, from which we have obtained the solitary wave, kink, and antikink solutions as special cases in Sec. 4. The conclusions are set out in Sec. 5 .

2. Formulation. We consider two semi-infinite, inviscid, incompressible magnetic fluids of densities $\rho_{1}$ and $\rho_{2}$ (see Fig. 1). The magnetic field $\mathbf{H}(0,0, H)$ acts normal to the interface, and the gravitational force per unit mass is $\mathbf{g}(0,0,-g)$. The body force in a magnetised medium can be expressed in the form [1]:

$$
\mathbf{f}=-\frac{1}{4 \pi} \nabla \int_{0}^{H}\left[\mu-\rho\left(\frac{\partial \mu}{\partial \rho}\right)_{H, T}\right] H d H+\frac{\mu}{\pi} \nabla H^{2},
$$

where $\rho$ is the density of the medium, and $\mu$ represents the magnetic permeability which we shall assume to be a constant. The basic equations governing the velocity potential $\Phi$ are

$$
\begin{array}{ll}
\nabla^{2} \Phi^{(1)}=0, & -\infty<z<\eta(x, t), \\
\nabla^{2} \Phi^{(2)}=0, & \eta(x, t)<z<\infty,
\end{array}
$$

where $\eta(x, t)$ is the elevation of the free surface measured from the unperturbed level.

As a result of perturbations, the distortion of the free surface yields an additional magnetic field $\mathbf{h}=-\nabla \psi(x, z)$, where the magnetic potential $\psi$ satisfies the equations

$$
\begin{aligned}
& \nabla^{2} \psi^{(1)}=0, \quad-\infty<z<\eta(x, t), \\
& \nabla^{2} \psi^{(2)}=0, \quad \eta(x, t)<z<\infty .
\end{aligned}
$$

Since the motion must vanish away from the interface, we must have

$$
\begin{array}{ccc}
\left|\nabla \Phi^{(1)}\right| \rightarrow 0, & \left|\nabla \psi^{(1)}\right| \rightarrow 0 & \text { as } z \rightarrow-\infty, \\
\left|\nabla \Phi^{(2)}\right| \rightarrow 0, & \left|\nabla \psi^{(2)}\right| \rightarrow 0 & \text { as } z \rightarrow \infty
\end{array}
$$

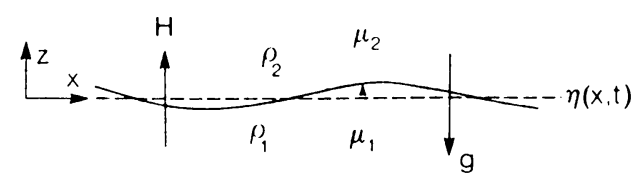

Fig. 1. The Basic Configuration 
At the free surface $z=\eta(x, t)$, the various boundary conditions are

$$
\begin{gathered}
\frac{\partial \eta}{\partial t}-\frac{\partial \Phi^{(j)}}{\partial z}+\frac{\partial \Phi^{(j)}}{\partial x} \frac{\partial \eta}{\partial x}=0, \quad(j=1,2) \\
\mu_{1}=H_{n}^{(1)}=\mu_{2} H_{n}^{(2)}, \\
H_{t}^{(1)}=H_{t}^{(2)} .
\end{gathered}
$$

Here, the normal and the tangential components of the magnetic field are represented by $H_{n}$ and $H_{t}$, respectively. The continuity of the normal stress across the interface $z=\eta(x, t)$ demands

$$
\begin{aligned}
\rho_{1} \frac{\partial \Phi^{(1)}}{\partial t}-\rho_{2} \frac{\partial \Phi^{(2)}}{\partial t} & +\left(\rho_{1}-\rho_{2}\right) g \eta+\frac{1}{2}\left(\rho_{1}\left(\nabla \Phi^{(1)}\right)^{2}-\rho_{2}\left(\nabla \Phi^{(2)}\right)^{2}\right) \\
& -T \frac{\partial^{2} \eta}{\partial x^{2}}\left(1+\left(\frac{\partial \eta}{\partial x}\right)^{2}\right)^{-3 / 2}=\frac{\mu-1}{8 \pi} H^{2}+\frac{(\mu-1)^{2}}{8 \pi} H_{n}^{2},
\end{aligned}
$$

where $T$ is the surface tension, $\mathbf{H}$ the uniform part of the magnetic field in the second medium and $\mu=\mu_{1} / \mu_{2}$.

As the system is conservative, the dispersive wave shall propagate in the medium. However, in the region $H \geqslant H_{c}$, the wave is capable of drawing energy from the potential energy of the system, thereby lettng the system operate through the most unstable mode $k=k_{c}$ (see Stuart [9]). To investigate the effects of spatial modulation in the neighbourhood of $\left(k_{c}, H_{c}\right)$, we employ the method of multiple scales, and introduce the variables $x_{n}=\varepsilon^{n} x, t_{n}=\varepsilon^{n} t$. The small parameter $\varepsilon=\left(H^{2} / H_{c}^{2}-1\right)^{1 / 2}$ defines how far the system departs from the bifurcation value $H_{c}$ (see Eckhaus [11]). In order to describe the nonlinear interactions of small but finite amplitude waves, we write

$$
\begin{gathered}
\Phi(x, z, t)=\sum_{n=1}^{2} \varepsilon^{n} \Phi_{n}+O\left(\varepsilon^{3}\right), \\
\psi(x, z, t)=\sum_{n=1}^{2} \varepsilon^{n} \psi_{n}+O\left(\varepsilon^{3}\right), \\
\frac{\partial}{\partial t}=\sum_{n=0}^{2} \varepsilon^{n} \frac{\partial}{\partial t_{n}}+O\left(\varepsilon^{3}\right), \\
\frac{\partial}{\partial x}=\sum_{n=0}^{2} \varepsilon^{n} \frac{\partial}{\partial x_{n}}+O\left(\varepsilon^{3}\right),
\end{gathered}
$$

and

$$
\eta(x, t)=\sum_{n=1}^{2} \varepsilon^{n} \eta_{n}+O\left(\varepsilon^{3}\right) .
$$

Here, $\Phi_{n}, \psi_{n}$, and $\eta_{n}$ are allowed to depend on the slower scales. To evaluate the boundary conditions (8) to (11), we use the Maclaurin's Series expansions at $z=0$ for the quantities 
involved. Then, substitution from equation (12) into the field equations (2) to (7) and the boundary conditions (8) to (11), and equating coefficients of equal powers in $\varepsilon$, we obtain the linear as well as successive higher order perturbation equations (see Appendix). The hierarchy of the equation for each order can be derived with the knowledge of the solutions for the previous order.

For the first order problem, the solutions are

$$
\begin{gathered}
\eta_{1}=A\left(x_{0}, x_{1} ; t_{0}, t_{1}\right) \exp (i \theta)+\text { c.c., } \\
\Phi_{1}^{(1)}=-\frac{i \omega}{k} A\left(x_{0}, x_{1} ; t_{0}, t_{1}\right) \exp (i \theta+k z)+\text { c.c., } \\
\Phi_{1}^{(2)}=\frac{i \omega}{k} A\left(x_{0}, x_{1} ; t_{0}, t_{1}\right) \exp (i \theta-k z)+\text { c.c., } \\
\psi_{1}^{(1)}=\frac{H}{\mu}\left(\frac{1-\mu}{1+\mu}\right) A \exp (i \theta+k z)+\text { c.c., } \\
\psi_{1}^{(2)}=-H\left(\frac{1-\mu}{1+\mu}\right) A \exp (i \theta-k z)+\text { c.c., }
\end{gathered}
$$

with

$$
\theta=k x_{0}-\omega t_{0} .
$$

The frequency $\omega$ and the wave number $k$ satisfy the dispersion relation:

$$
\frac{\omega^{2}}{k}\left(\rho_{1}+\rho_{2}\right)=\left(\rho_{1}-\rho_{2}\right) g+T k^{2}-\frac{(\mu-1)^{2} H^{2}}{4 \pi \mu(\mu+1)} k .
$$

By letting $\omega=0$ in the equation (19), the neutral stability curve can be obtained. The critical value $H_{c}$ of the magnetic field is furnished by (19) when $k_{c}=\left[\left(\rho_{1}-\rho_{2}\right) g / T\right]^{1 / 2}$ :

$$
H_{c}^{2}=\frac{8 \pi \mu(\mu+1)}{(\mu-1)^{2}}\left[\left(\rho_{1}-\rho_{2}\right) g T\right]^{1 / 2} .
$$

It is clear that the system is unstable for $H \geqslant H_{c}$.

In the next section we show that within the context of the nonlinear theory, the system bifurcates to a new state in which the unsteady motion leads to the soft and the hard modes of excitation.

3. Nonlinear Klein-Gordon equation. To obtain the envelope equation, we solve the second order problem. The bounded solutions for $k=k_{c}$ and $H=H_{c}$ are found as

$$
\begin{gathered}
\Phi_{2}^{(1)}=\frac{1}{k} \frac{\partial A}{\partial t_{1}} \exp \left(i \theta_{0}+k_{c} z\right)+\text { c.c., } \\
\Phi_{2}^{(2)}=\frac{1}{k} \frac{\partial A}{\partial t_{1}} \exp \left(i \theta_{0}-k_{c} z\right), \\
\psi_{2}^{(1)}=\frac{i H}{k \mu}\left(\frac{1-\mu}{1+\mu}\right)(1-z k) \frac{\partial A}{\partial x_{1}} \exp \left(i \theta_{0}+k_{c} z\right) \\
+\left(\Lambda-k_{c}\right) \frac{H(1-\mu)}{\mu(1+\mu)} A^{2} \exp \left(2 i \theta_{0}+2 k_{c} z\right)+\text { c.c. }
\end{gathered}
$$




$$
\begin{gathered}
\psi_{2}^{(2)}=-\frac{i H(1-\mu)}{k(1+\mu)}(1+z k) \frac{\partial A}{\partial x_{1}} \exp \left(i \theta_{0}-k_{c} z\right) \\
-\left(\Lambda+k_{c}\right) \frac{H(1-\mu)}{(1+\mu)} A^{2} \exp \left(2 i \theta-2 k_{c} z\right)+\text { c.c. } \\
\eta_{2}=\lambda A^{2} \exp \left(2 i \theta_{0}\right)+\text { c.c. }
\end{gathered}
$$

where

$$
\Lambda=\frac{k_{c}^{2} v^{2}((\mu-1) /(\mu+1))}{\left[\left(\rho_{1}-\rho_{2}\right) g+4 k_{c}^{2} T-2 v^{2} k_{c}\right]},
$$

and

$$
\theta_{0}=k_{c} x_{0} \text {. }
$$

In deriving the solutions given by the equations (21) to (27), we have made use of the non-secularity condition for $\eta_{2}$, i.e.,

$$
i \frac{\partial A}{\partial x_{1}}\left[\frac{(\mu-1)^{2} H_{c}^{2}}{4 \pi \mu(\mu+1)}-2 T k\right]_{k=k_{c}}=0
$$

which implies non vanishing $\partial A / \partial x_{1}$. It is interesting to observe that in our solutions for $\psi_{2}^{(1)}$ and $\psi_{2}^{(2)}$, given by the equations (23) and (24), there is no contribution due to the constant term $|A|^{2}$ as claimed by Twombly and Thomas [8] in their solutions. However, our results do agree with those of Zaitsev and Shliomis [18].

On substituting the first and second order solutions already obtained into the equations governing the third order problem in $\varepsilon$, we get the following equation for the slowly varying amplitude:

$$
\frac{\partial^{2} A}{\partial t_{1}^{2}}-\Omega^{2} \frac{\partial^{2} A}{\partial x_{1}^{2}}=\sigma A+Q|A|^{2} A
$$

where

$$
\begin{gathered}
\Omega^{2}=\frac{\left(\rho_{1}-\rho_{2}\right) g}{k\left(\rho_{1}+\rho_{2}\right)}, \\
\sigma=2 \frac{\left(\rho_{1}-\rho_{2}\right) g k}{\left(\rho_{1}+\rho_{2}\right)}\left(\frac{H^{2}}{H_{c}^{2}}-1\right),
\end{gathered}
$$

and

$$
Q=\frac{2\left(\rho_{1}-\rho_{2}\right) g k^{3}}{4(\mu+1)^{2}\left(\rho_{1}+\rho_{2}\right)}\left(11\left(\mu^{2}+1\right)-42 \mu\right) .
$$

The equation (29) is the well known nonlinear Klein-Gordon equation which is encountered in other important applications such as the buckling problem by Lang and Newell [12], the baroclinic instability by Pedlosky [13], and the Kelvin-Helmholtz instability by Weissman [14].

For real $A$, the equation (29) admits a solution in terms of the Jacobian elliptic function. The character of the solution depends sensitively on the sign of the coefficients $\sigma$ and $Q$. 
The interaction parameter $Q$ is positive for $\mu<\mu_{c}(=3.54)$ and negative for $\mu>\mu_{c}$, thus leading to the soft and the hard modes of excitation, respectively.

We shall now examine the various cases of physical interest with $\Omega=0$.

(i) When $\left(H^{2} / H_{c}^{2}-1\right)$ and $Q$ are both negative, we observe from equation (29) that the nonlinearity generates a stabilizing influence leading to the bounded solutions. The phase plane anlaysis shows that the trajectories are closed curves representing the $c n$ function.

(ii) When $\left(H^{2} / H_{c}^{2}-1\right)$ is negative and $Q$ is positive, we have hard excitations. The two terms on the right-hand side of the equation (29) have opposite sense, the first or second being greater or less in magnitude according to whether the amplitude $A$ is less than or greater than $A_{e}$, where

$$
A_{e}=2^{1 / 2} k_{c}^{-1}\left(\frac{H^{2}}{H_{c}^{2}}-1\right)\left(42 \mu-11\left(\mu^{2}+1\right)\right) .
$$

In this case, $A_{e}$ is a threshold value in the sense that if the initial amplitude $A_{0}<A_{e}$, the solution is bounded as $t \rightarrow \infty$, and if $A_{0}>A_{e}$, the solution breaks down after a finite time. This kind of solution is represented in the Fig. 2. The inclusion of the higher order terms results in a rapid transition to the turbulence. Such a case is termed "subcritical" instability since the instability may occur with finite amplitude $A>A_{e}$ when all the small perturbations are stable as predicted by the linear theory.

(iii) When $\left(H^{2} / H_{c}^{2}-1\right)>0$ and $Q<0$, the equation (29) upon integration yields

$$
\left(\frac{\partial A}{\partial t}\right)^{2}=c+\sigma A^{2}+\frac{1}{2} Q A^{4}
$$

where $c$ is a constant of integration.

Assuming the initial conditions as $A(0)=A_{0}$ and $(\partial A / \partial t)_{t=0}=B_{0}$, we get

$$
c=B_{0}^{2}-\sigma A_{0}^{2}-\frac{Q}{2} A_{0}^{4} \text {. }
$$

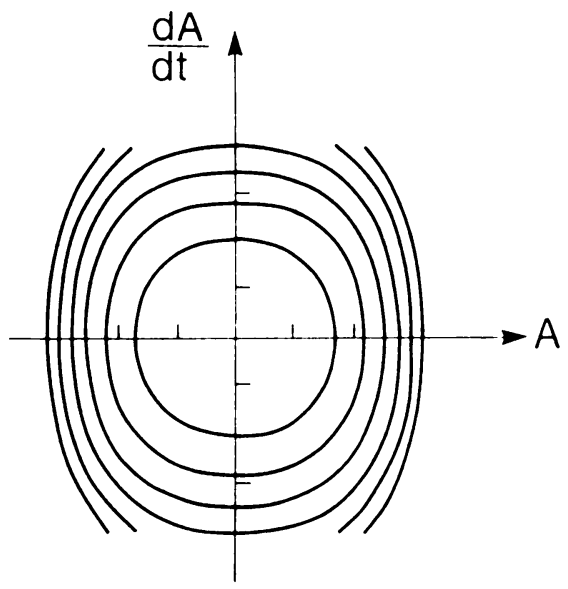

Fig. 2. The phase plot of $\frac{d A}{d t}$ and $A$ with $\left(\frac{H^{2}}{H_{c}^{2}}-1\right)<0$ and $\mu<\mu_{c}$. 
The phase diagram is sketched in the Fig. 3. We note that there are three equilibrium states in the phase plot: one at the origin, and two at the points $A= \pm A_{e}$. These states correspond to the bifurcation of equilibrium, and are not dynamically conducted with one another so that neither of them can be reached by the others through the evolution of the instability. For $c=0$, the amplitude $A(t)$ grows exponentially, passes through the maximum at $A_{m}^{2}=-2 \sigma / Q$, and finally decays exponentially to zero. This situation is remarkably different from the one encountered in the case of the Landau equation in fluid mechanics which exhibits amplitude saturation [9]. In our solution, the nonlinear stabilization is in the form of non-sinusoidal periodic motions without giving amplitude saturation as reported earlier $[7,15]$.

(iv) When $\left(H^{2} / H_{c}^{2}-1\right)>0$ and $Q>0$, both the terms on the right hand side of the equation (29) are positive. The amplitude $A(t)$, therefore, increases super-exponentially. This situation is often termed as "explosive" instability. The solution breaks down after a finite time. In other words, there is a faster transition from the coherent motion to the turbulence in this case. In fact, this kind of breakdown may not occur in practice. However, this does point out the limitations of the formulation developed in this paper, and indicates the desirability of including the dispersive effects, dissipation and terms of order higher than three in powers of $A$ in the derivation of the equation (29).

Special cases. We now consider the progressive wave solution, taking into account the effects of modulation in both space and time. We introduce the new variables

$$
\begin{aligned}
\zeta & =x_{1}-c t_{1}, \quad X=\left(\frac{|\sigma|^{1 / 2}}{\Omega}\right) x_{1}, \quad T=|\sigma|^{1 / 2} t_{1} \\
A^{\prime} & =-\left(\frac{|Q|}{|\sigma|}\right)^{1 / 2} A, \quad C^{\prime}=\frac{c}{\Omega} .
\end{aligned}
$$

Substituting from (35) and dropping primes for convenience, the different solutions of the equation (29) are possible depending on the sign of $c^{2}-\Omega^{2}, \sigma$, and $Q$. We shall investigate only those cases where the linear instability is saturated by nonlinear effects. With $\left(H^{2} / H_{c}^{2}-1\right)>0$ and $Q<0$, i.e. the case of soft excitations, equation (29) becomes

$$
\left(C^{2}-1\right) \frac{d^{2} A}{d \zeta^{2}}=A-A^{3} \text {. }
$$

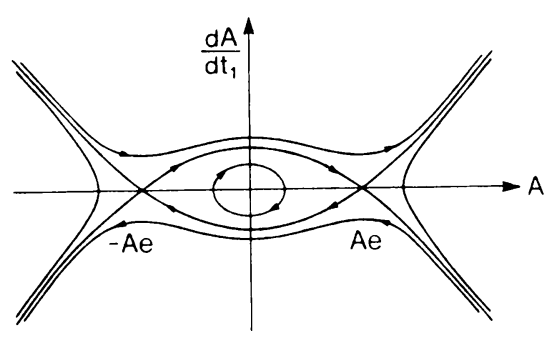

Fig. 3. The phase plot of $\frac{d A}{d t}$ and $A$ with $\left(\frac{H^{2}}{H_{c}^{2}}-1\right)<0$ and $\mu>\mu_{c}$ 
The above equation (36) yields different solutions depending on the sign of $\left(C^{2}-1\right)$. When $C^{2}>1$, the speed of the wave is greater than the group velocity of the wave packet. We should remark here that Whitham [16] studied the side-band instability for the nonlinear Klein-Gordon equation (29). His analysis revealed that the carrier wave is unstable to the side-band perturbations, and results in the growth of the modulations.

In our presentation, however, the development of large modulations leads to the bell shaped soliton whose amplitude is given by

$$
A=(2)^{1 / 2} \operatorname{sech}\left[\Delta^{-1}(X-C T)\right],
$$

where

$$
\Delta=\left(C^{2}-1\right)^{1 / 2}
$$

From equations (37) and (38), we observe that the soliton width $\Delta$ is entirely determined by $C$ in such a manner that the greater the width, the faster the soliton propagates. For the case when $C^{2}<1$, the equation (36) admits the solution

$$
A= \pm \tanh \left[\delta^{-1}(X-C T)\right] \text {, }
$$

where

$$
\delta=\left[2\left(1-C^{2}\right)\right]^{1 / 2} .
$$

This solution is in the form of a kink (see Makhankov [17]), which has different boundary conditions at $X= \pm \infty$. The solution with the plus sign in (39) is called "kink" and that with the minus sign is called the "anti-kink". The "kink" and "anti-kink" are solitary waves rather than solitons because of their inability to survive collisions. It should also be noted here that for the case $\left(H^{2} / H_{c}^{2}-1\right)<0, Q>0$, i.e. hard excitations case, we obtain bell shaped solitons for $C^{2}<1$, and kink solutions for $C^{2}>1$.

5. Conclusions. We have determined the weakly nonlinear solutions which describe the nonlinear evolution of wave pocket in two superposed magnetic fluids in the presence of the transverse magnetic field. The growth of the instability amplitude is governed by a nonlinear Klein-Gordon equation. If the spatial effects are ignored, we can get subcritical, supercritical, and explosive instabilities, depending upon the signs of $\left(H^{2} / H_{c}^{2}-1\right)$ and the interaction parameter $Q$. The combined effects of the temporal and spatial modulations give rise to the bell shaped soliton and the kink solutions.

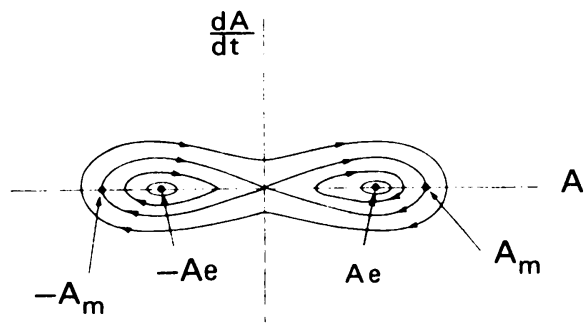

Fig. 4. The phase plot of $\frac{d A}{d t}$ and $A$ with $\left(\frac{H^{2}}{H_{c}^{2}}-1\right)>0$ and $\mu<\mu_{c}$ 


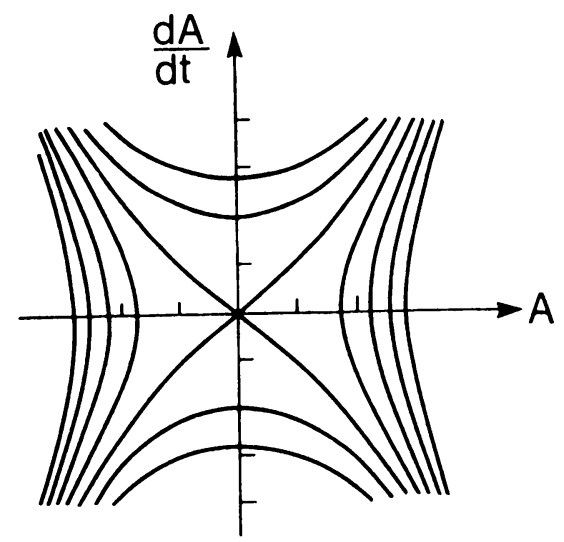

Fig. 5. The phase plot of $\frac{d A}{d t}$ and $A$ with $\left(\frac{H^{2}}{H_{c}^{2}}-1\right)>0$ and $\mu>\mu_{c}$.

Appendix. The various operators are defined as follows:

$$
\begin{gathered}
L_{1}=\frac{\partial^{2}}{\partial x_{0}^{2}}+\frac{\partial^{2}}{\partial z^{2}}, \quad L_{2}\left(\eta, \phi_{1}^{(j)}\right)=\frac{\partial \eta}{\partial t_{0}}-\frac{\partial_{1}^{(j)}}{\partial z}, \\
L_{3}\left(\psi_{1}^{(1)}, \psi_{2}^{(2)}\right)=\mu \frac{\partial \psi_{1}^{(1)}}{\partial z}-\frac{\partial \psi_{2}^{(2)}}{\partial z}, \\
L_{4}\left(\psi_{1}^{(1)}, \psi_{2}^{(2)}, \eta\right)=\frac{\partial \psi_{1}^{(1)}}{\partial x_{0}}-\frac{\partial \psi_{2}^{(2)}}{\partial x_{0}}-\frac{(\mu-1) H}{\mu} \frac{\partial \eta}{\partial x_{0}}, \\
L_{5}\left(\phi_{1}^{(1)}, \phi_{2}^{(2)}, \eta, \psi\right)=\rho_{1} \frac{\partial \phi_{1}^{(1)}}{\partial t_{0}}-\rho_{2} \frac{\partial}{\partial t_{2}} \phi_{2}^{(2)}+\left(\rho_{1}-\rho_{2}\right) g \eta-T \frac{\partial^{2} \eta}{\partial x_{0}^{2}} \\
+\frac{(\mu-1)}{4 \pi} H \frac{\partial \psi}{\partial z} .
\end{gathered}
$$

The equations governing the first order theory are

$$
\begin{aligned}
& L_{1}\left(\phi_{1}^{(j)}\right)=0, \\
& L_{1}\left(\psi_{1}^{(j)}\right)=0,
\end{aligned}
$$

and the corresponding boundary conditions at $z=0$ as

$$
\begin{gathered}
L_{2}\left(\eta_{1}, \phi_{1}^{(j)}\right)=0, \\
L_{3}\left(\psi_{1}^{(1)}, \psi_{1}^{(2)}\right)=0, \\
L_{4}\left(\psi_{1}^{(1)}, \psi_{1}^{(2)}, \eta_{1}\right)=0, \\
L_{5}\left(\phi_{1}^{(1)}, \phi_{1}^{(2)}, \eta_{1}, \psi_{1}^{(1)}\right)=0 .
\end{gathered}
$$


The second order theory is given by

$$
\begin{aligned}
& L_{1}\left(\phi_{2}^{(j)}\right)=-2 \frac{\partial^{2} \phi_{1}^{(j)}}{\partial x_{0} \partial x_{1}}, \\
& L_{1}\left(\psi_{2}^{(j)}\right)=-2 \frac{\partial^{2}\left(\psi_{1}^{(j)}\right)}{\partial x_{0} \partial x_{1}},
\end{aligned}
$$

and the boundary conditions at $z=0$ as

$$
\begin{gathered}
L_{2}\left(\eta_{2}, \phi_{2}^{(j)}\right)=-\frac{\partial \eta_{1}}{\partial t_{1}}+\eta_{1} \frac{\partial^{2} \phi_{1}^{(j)}}{\partial z^{2}}-\frac{\partial \phi_{1}^{(j)}}{\partial x_{0}} \frac{\partial \eta_{1}}{\partial x_{0}}, \\
L_{3}\left(\psi_{2}^{(1)}, \psi_{2}^{(2)}\right)=-\eta_{1}\left(\mu \frac{\partial^{2} \psi_{1}^{(1)}}{\partial z^{2}}-\frac{\partial^{2} \psi_{1}^{(2)}}{\partial z^{2}}\right)+\left(\frac{\partial \eta_{1}}{\partial x_{0}}\right)\left(\mu \frac{\partial \psi_{1}^{(1)}}{\partial x_{0}}-\frac{\partial \psi_{1}^{(2)}}{\partial x_{0}}\right), \\
L_{4}\left(\psi_{2}^{(1)}, \psi_{2}^{(2)}, \eta_{2}\right)=-\eta_{1}\left(\frac{\partial^{2} \psi_{1}^{(1)}}{\partial x_{0} \partial z}-\frac{\partial^{2} \psi_{1}^{(2)}}{\partial x_{0} \partial z}\right)-\frac{\partial \eta_{1}}{\partial x_{0}}\left(\frac{\partial \psi_{1}^{(1)}}{\partial z}-\frac{\partial \psi_{1}^{(2)}}{\partial z}\right) \\
-\left(\frac{\partial \eta_{1}}{\partial x_{1}}\right) \frac{H}{\mu}(1-\mu)-\left(\frac{\partial \psi_{1}^{(1)}}{\partial x_{1}}-\frac{\partial \psi_{1}^{(2)}}{\partial x_{1}}\right),
\end{gathered}
$$

$L_{5}\left(\phi_{2}^{(1)}, \phi_{2}^{(2)}, \eta_{2}, \psi_{2}^{(1)}\right)$

$$
\begin{gathered}
=-\rho_{1} \frac{\partial \phi_{1}^{(1)}}{\partial t_{1}} \rho_{2} \frac{\partial \phi_{1}^{(2)}}{\partial t_{1}}-\frac{\rho_{1}}{2}\left(\frac{\partial \phi_{1}^{(1)}}{\partial x_{0}}\right)^{2}-\frac{\rho_{1}}{2}\left(\frac{\partial \phi_{1}^{(1)}}{\partial z}\right)^{2} \\
+\frac{\rho_{2}}{2}\left(\frac{\partial \phi_{1}^{(2)}}{\partial x_{0}}\right)^{2}+\frac{\rho_{2}}{2}\left(\frac{\partial \phi_{1}^{(2)}}{\partial z}\right)^{2}-\eta_{1} \rho_{1} \frac{\partial^{2} \phi_{1}^{(1)}}{\partial t_{0} \partial z}+\rho_{2} \eta_{2} \frac{\partial^{2} \phi_{1}^{(2)}}{\partial t_{0} \partial z} \\
+2 T \frac{\partial^{2} \eta_{1}}{\partial x_{0} \partial z}+\frac{(\mu-1)}{8 \pi}\left[\left(\frac{\partial \psi_{1}^{(1)}}{\partial x_{0}}\right)^{2}+\mu\left(\frac{\partial \psi_{1}^{(1)}}{\partial z}\right)^{2}+\left(\frac{\partial \eta_{1}}{\partial x_{0}}\right)^{2} \frac{H^{2}}{\mu^{2}}(1-\mu)\right. \\
\left.-2 H \eta_{1} \frac{\partial^{2} \psi_{1}^{(1)}}{\partial z^{2}}+2 H \frac{\partial \eta_{1}}{\partial x_{0}} \frac{\partial \psi_{1}^{(1)}}{\partial x_{0}}\left(\frac{\mu-1}{\mu}\right)\right] .
\end{gathered}
$$

For the third order problem, we have

$$
\begin{aligned}
& L_{1}\left(\phi_{3}^{(j)}\right)=-2 \frac{\partial^{2} \phi_{1}^{(j)}}{\partial x_{0} \partial x_{2}}-2 \frac{\partial^{2} \phi_{2}^{(j)}}{\partial x_{0} \partial x_{1}}-\frac{\partial^{2} \phi_{1}^{(j)}}{\partial x_{1}^{2}}, \\
& L_{1}\left(\psi_{3}^{(j)}\right)=-2 \frac{\partial \psi_{1}^{(j)}}{\partial x_{0} \partial x_{2}}-2 \frac{\partial^{2} \psi_{2}^{(j)}}{\partial x_{0} \partial x_{1}}-\frac{\partial^{2} \psi_{1}^{(j)}}{\partial x_{1}^{2}},
\end{aligned}
$$

subject to the boundary conditions at $z=0$ :

$$
\begin{aligned}
L_{2}\left(\eta_{3}, \phi_{3}^{(j)}\right)= & -\frac{\partial \eta_{1}}{\partial t_{2}} \frac{\partial \eta_{2}}{\partial t_{1}}+\eta_{1} \frac{\partial^{2} \phi_{2}^{(j)}}{\partial z^{2}}+\eta_{2} \frac{\partial^{2} \phi_{1}^{(j)}}{\partial z^{2}}+\frac{1}{2} \eta_{1}^{2} \frac{\partial^{3} \phi_{1}^{(j)}}{\partial z^{3}} \\
& -\frac{\partial \phi_{1}^{(j)}}{\partial x_{0}} \frac{\partial \eta_{2}}{\partial x_{0}}-\frac{\partial \phi_{2}^{(j)}}{\partial x_{0}} \frac{\partial \eta_{1}}{\partial x_{0}}-\eta_{1} \frac{\partial^{2} \phi_{2}^{(j)}}{\partial x_{0} \partial z} \frac{\partial \eta_{1}}{\partial x_{0}} \\
& -\frac{\partial \phi_{1}^{(j)}}{\partial x_{1}} \frac{\partial \eta_{1}}{\partial x_{0}}-\frac{\partial \phi_{1}^{(j)}}{\partial x_{0}} \frac{\partial \eta_{1}}{\partial x_{1}}
\end{aligned}
$$




$$
\begin{aligned}
& L_{3}\left(\psi_{3}^{(1)}, \psi_{3}^{(2)}\right)=-\eta_{1}\left(\mu \frac{\partial^{2} \psi_{2}^{(1)}}{\partial z^{2}}-\frac{\partial^{2} \psi_{2}^{(2)}}{\partial z^{2}}\right)-\eta_{2}\left(\mu \frac{\partial^{2} \psi_{2}^{(1)}}{\partial z^{2}}-\frac{\partial^{2} \psi_{1}^{(2)}}{\partial z^{2}}\right) \\
& -\frac{\eta_{1}^{2}}{2}\left(\mu \frac{\partial^{3} \psi_{1}^{(1)}}{\partial z^{3}}-\frac{\partial^{3} \psi_{1}^{(2)}}{\partial z^{3}}\right)+\left(\frac{\partial \eta_{1}}{\partial x_{0}}\right)\left(\mu \frac{\partial \psi_{2}^{(1)}}{\partial x_{0}}-\frac{\partial \psi_{2}^{(2)}}{\partial x_{0}}\right) \\
& +\left(\frac{\partial \eta_{2}}{\partial x_{0}}\right)\left(\mu \frac{\partial \psi_{1}^{(1)}}{\partial x_{0}}-\frac{\partial \psi_{l}^{(2)}}{\partial x_{0}}\right)+\left(\frac{\partial \eta_{1}}{\partial x_{0}}\right)\left(\mu \eta_{1} \frac{\partial^{2} \psi_{l}^{(1)}}{\partial x_{0} \partial z}-\eta_{1} \frac{\partial^{2} \psi_{l}^{(2)}}{\partial x_{0} \partial z}\right) \\
& +\left(\frac{\partial \eta_{1}}{\partial x_{0}}\right)\left(\mu \frac{\partial \psi_{1}^{(1)}}{\partial x_{0}}-\frac{\partial \psi_{1}^{(2)}}{\partial x_{0}}\right)+\left(\frac{\partial \eta_{1}}{\partial x_{0}}\right)\left(\mu \frac{\partial \psi_{1}^{(1)}}{\partial x_{1}}-\frac{\partial \psi_{1}^{(2)}}{\partial x_{1}}\right), \\
& L_{4}\left(\psi_{3}^{(1)}, \psi_{3}^{(2)}, \eta_{3}\right)=-\eta_{1}\left(\frac{\partial^{2} \psi_{2}^{(1)}}{\partial x_{0} \partial z}-\frac{\partial^{2} \psi_{2}^{(2)}}{\partial x_{0} \partial z}\right)-\eta_{2}\left(\frac{\partial^{2} \psi_{1}^{(1)}}{\partial x_{0} \partial z}-\frac{\partial^{2} \psi_{1}^{(2)}}{\partial x_{0} \partial z}\right) \\
& -\frac{\eta_{1}^{2}}{2}\left(\frac{\partial^{3} \psi_{1}^{(1)}}{\partial x_{0} \partial z^{2}}-\frac{\partial^{3} \psi_{1}^{(2)}}{\partial x_{0} \partial z^{2}}\right)-\left(\frac{\partial \eta_{1}}{\partial x_{0}}\right)\left(\frac{\partial \psi_{2}^{(1)}}{\partial z}-\frac{\partial \psi_{2}^{(2)}}{\partial z}\right) \\
& -\left(\frac{\partial \eta_{2}}{\partial x_{0}}\right)\left(\frac{\partial \psi_{1}^{(1)}}{\partial z}-\frac{\partial \psi_{1}^{(2)}}{\partial z}\right) \\
& -\eta_{1}\left(\frac{\partial \eta_{1}}{\partial x_{0}}\right)\left(\frac{\partial^{2} \psi_{1}^{(1)}}{\partial z^{2}}-\frac{\partial^{2} \psi_{1}^{(2)}}{\partial z^{2}}\right)-\left(\frac{\partial \psi_{2}^{(1)}}{\partial x_{1}}-\frac{\partial \psi_{2}^{(2)}}{\partial x_{1}}\right) \\
& -\left(\frac{\partial \psi_{1}^{(1)}}{\partial x_{2}}-\frac{\partial \psi_{1}^{(2)}}{\partial x_{2}}\right)+\left(\frac{1-\mu}{\mu}\right) H\left(\frac{\partial \eta_{2}}{\partial x_{1}}+\frac{\partial \eta_{1}}{\partial x_{2}}\right) \\
& -\left(\frac{\partial \eta_{1}}{\partial x_{1}}\right)\left(\frac{\partial \psi_{1}^{(1)}}{\partial z}-\frac{\partial \psi_{1}^{(2)}}{\partial z}\right)
\end{aligned}
$$

$$
\begin{aligned}
L_{5}\left(\phi_{3}^{(1)}, \phi_{3}^{(2)}, \eta_{3}, \psi_{3}^{(1)}\right)= & -\rho_{1} \frac{\partial \phi_{1}^{(1)}}{\partial t_{2}}-\rho_{1} \frac{\partial \phi_{2}^{(1)}}{\partial t_{1}}+\rho_{2} \frac{\partial \phi_{1}^{(2)}}{\partial t_{2}}+\rho_{1} \frac{\partial \phi_{2}^{(1)}}{\partial t_{1}} \\
& +2 \frac{\partial^{2} \eta_{1}}{\partial x_{0} \partial x_{2}}-\rho_{1} \frac{\partial \phi_{1}^{(1)}}{\partial x_{0}} \frac{\partial \phi_{2}^{(1)}}{\partial x_{0}}-\rho_{1} \eta_{1} \frac{\partial \phi_{1}^{(1)}}{\partial x_{0}} \frac{\partial^{2} \phi_{1}^{(1)}}{\partial x_{0} \partial z} \\
& +\rho_{2} \frac{\partial \phi_{1}^{(2)}}{\partial x_{0}} \frac{\partial \phi_{1}^{(2)}}{\partial x_{0}}+\rho_{2} \eta_{1} \frac{\partial \phi_{1}^{(2)}}{\partial x_{0}} \frac{\partial^{2} \phi_{1}^{(2)}}{\partial x_{0} \partial z}-\rho_{1} \frac{\partial \phi_{1}^{(1)}}{\partial z} \frac{\partial \phi_{2}^{(1)}}{\partial z} \\
& -\rho_{1} \eta_{1} \frac{\partial \phi_{1}^{(1)}}{\partial z} \frac{\partial^{2} \phi_{1}^{(1)}}{\partial z^{2}}+2 T \frac{\partial^{2} \eta_{1}}{\partial x_{0} \partial x_{2}}+2 T \frac{\partial^{2} \eta_{2}}{\partial x_{0} \partial x_{1}}+T \frac{\partial^{2} \eta_{1}}{\partial x_{1}^{2}} \\
& -\rho_{1} \frac{\partial \phi_{1}^{(1)}}{\partial x_{0}} \frac{\partial \phi_{1}^{(1)}}{\partial x_{1}}+\rho_{2} \frac{\partial \phi_{1}^{(2)}}{\partial x_{0}} \frac{\partial \phi_{1}^{(2)}}{\partial x_{1}}+\rho_{2} \frac{\partial \phi_{1}^{(2)}}{\partial z} \frac{\partial \phi_{2}^{(2)}}{\partial z} \\
& +\rho_{2} \eta_{1} \frac{\partial \phi_{1}^{(2)}}{\partial z} \frac{\partial^{2} \phi_{1}^{(2)}}{\partial z^{2}}-\rho_{1} \eta_{1} \frac{\partial^{2} \phi_{1}^{(1)}}{\partial t_{0} \partial z}-\rho_{1} \eta_{1} \frac{\partial^{2} \phi_{1}^{(2)}}{\partial t_{0} \partial z} \\
& -\frac{\rho_{1}}{2} \eta_{1}^{2} \frac{\partial^{3} \phi_{1}^{(1)}}{\partial t_{0} \partial z^{2}}+\rho_{2} \eta_{2} \frac{\partial^{2} \phi_{1}^{(2)}}{\partial t_{0} \partial z}+\rho_{2} \eta_{1} \frac{\partial^{2} \phi_{2}^{(2)}}{\partial t_{0} \partial z}+\frac{1}{2} \rho \eta_{1}^{2} \frac{\partial^{3} \phi_{2}^{(1)}}{\partial t_{0} \partial z^{2}}
\end{aligned}
$$




$$
\begin{aligned}
& -\rho_{1} \eta_{2} \frac{\partial^{2} \phi_{1}^{(1)}}{\partial t_{1} \partial z}+\rho_{2} \eta_{1} \frac{\partial^{2} \phi_{1}^{(2)}}{\partial t_{1} \partial z}-\frac{3}{2} \frac{\partial^{2} \eta_{1}}{\partial x_{0}^{2}}\left(\frac{\partial \eta_{1}}{\partial x_{0}}\right)^{2} \\
& +\frac{(\mu-1)}{8 \pi}\left[2 \frac{\partial \psi_{1}^{(1)}}{\partial x_{0}} \frac{\partial \psi_{2}^{(1)}}{\partial x_{0}}+2 \eta_{1} \frac{\partial \psi_{1}^{(1)}}{\partial x_{0}} \frac{\partial^{2} \psi_{1}^{(1)}}{\partial x_{0} \partial z}\right. \\
& +2 \mu\left(\frac{\partial \psi_{1}^{(1)}}{\partial z} \frac{\partial \psi_{2}^{(1)}}{\partial z}+\eta_{1} \frac{\partial \psi_{1}^{(1)}}{\partial z} \frac{\partial^{2} \psi_{1}^{(1)}}{\partial z^{2}}\right) \\
& -2(\mu-1) \frac{\partial \eta_{1}}{\partial x_{0}} \frac{\partial \psi_{1}^{(1)}}{\partial x_{0}} \frac{\partial \psi_{1}^{(1)}}{\partial z}+2 \frac{H^{2}}{\mu^{2}}(1-\mu) \frac{\partial \eta_{1}}{\partial x_{0}} \frac{\partial \eta_{2}}{\partial x_{0}}+\frac{2 H}{\mu}(\mu-1) \\
& \quad \times\left(\frac{\partial \eta_{1}}{\partial x_{0}} \frac{\partial \psi_{2}^{(1)}}{\partial x_{0}}+\frac{\partial \eta_{2}}{\partial x_{0}} \frac{\partial \psi_{1}^{(1)}}{\partial x_{0}}+\eta_{1} \frac{\partial \eta_{1}}{\partial x_{0}} \frac{\partial^{2} \psi_{1}}{\partial x_{0} \partial z}\right) \\
& -2 H\left(\eta_{1} \frac{\partial^{2} \psi_{2}^{(1)}}{\partial z^{2}}+\frac{1}{2} \eta_{1}^{3} \frac{\partial^{3} \psi_{1}^{(1)}}{\partial z^{3}}+\eta_{2} \frac{\partial^{2} \psi_{1}}{\partial z^{2}}\right) \\
& +\frac{2 H}{\mu}\left(\frac{\partial \eta_{1}}{\partial x_{0}}\right)^{2}\left(\frac{\partial \psi_{1}^{(1)}}{\partial z}\right)(\mu-1)+2 \frac{\partial \psi_{1}}{\partial x_{1}} \frac{\partial \psi_{2}}{\partial x_{0}}+2 \frac{H^{2}}{\mu^{2}}(1-\mu) \frac{\partial \eta_{1}}{\partial x_{0}} \frac{\partial \eta_{1}}{\partial x_{1}} \\
& \left.+2 \frac{H(\mu-1)}{\mu}\left(\frac{\partial \eta_{1}}{\partial x_{0}} \frac{\partial \psi_{1}}{\partial x_{1}}+\frac{\partial \eta_{1}}{\partial x_{1}} \frac{\partial \psi_{1}}{\partial x_{0}}\right)\right]
\end{aligned}
$$

\section{REFERENCES}

[1] M. D. Cowley and R. E. Rosensweig, The interfacial instability of a ferromagnetic fluid, J. Fluid Mech. 30 , 671 (1967)

[2] R. E. Rosensweig, Pattern formation in magnetic fluids, Fourth Internat. Conf. on Synergetics, Ed. by H. Haken, Springer-Verlag, Berlin (1983)

[3] R. E. Zelazo and J. R. Melcher, Dynamics and stability of ferro-fluids, J. Fluid Mech. 39, 1 (1969)

[4] M. I. Shlimois and Yu. L. Raikher, Experimental investigations of magnetic fluids, I.E.E.E. Trans. Mag. 16, $237(1980)$

[5] A. Gailitis, Formation of the hexagonal pattern on the surface of a ferromagnetic fluid in an applied magnetic field, J. Fluid Mech. 82, 401 (1977)

[6] E. A. Kuznetsov and M. D. Spektor, Existence of a hexagonal relief on the surface of a dielectric fluid in an external electric field, Sov. Phys. JETP 44, 136 (1976)

[7] J. P. Brancher, Waves and instabilities on a plane interface between ferrofluids and nonmagnetic fluids, Thermo-mechanics $s^{s}$ of Magnetic Fluids, Ed. by B. Berkovsky, Hemisphere Publishing Corp. (1977)

[8] E. Twombly and J. W. Thomas, Theory of non-linear waves on the surface of a magnetic fluid, I.E.E.E. Trans. on Magnetics 16, 214 (1980)

[9] J. T. Stuart, Bifurcation theory in Non-linear Hydrodynamic Stability, Application of Bifurcation Theory, Ed. by P. H. Rabinowitz, New York, Academic Press (1977)

[10] A. C. Newell, Envelope equation, Lect. Appl. Math. 15, 157 (1974)

[11] W. Eckhaus, Studies in nonlinear stability theory, Springer (1965)

[12] C. G. Lang and A. C. Newell, The post-buckling problem for thin elastic shells, SIAM J. Appl. Math. 21, 605 (1971)

[13] J. Pedlosky, The finite Amplitude Dynamics of Baroclinic Waves, Application of Bifurcation Theory, Ed. by P. H. Rabinowitz, New York, Academic Press (1977) 
[14] M. A. Weissman, Nonlinear wave packets in the kelvin-helmholtz instability, Phil. Trans. Roy. Soc. London 290, 58 (1979)

[15] S. K. Malik and M. Singh, Nonlinear instability in superposed magnetic fluids, Third Internat. Conf. on Magnetic Fluids (1983)

[16] G. B. Whitham, Linear and nonlinear waves, Wiley, New York (1974)

[17] V. G. Makhankov, The dynamics of classical solitons, Phys. Rep. 35C, I (1978)

[18] V. M. Zaitsev and M. I. Shliomis, The nature of the instability of the interface between two liquids in a constant field, Dokl. Akad. Nauk SSR 188, 1261 (1969) 